However, some resources are not fully utilised such as KIT days and a 'supervised-on-call' period. Increased awareness of the policy and implementation of RTT meetings is required alongside increasing accessibility of training-resources to enable appropriate tailored support for returning-trainees.

\section{G403(P) MOODSEY: DEVELOPING A THEMATIC REAL-TIME STAFF MORALE TRACKING MODEL}

${ }^{1,2} \mathrm{R}$ Daniels, ${ }^{2} \mathrm{R}$ Bhatt, ${ }^{2} \mathrm{~S}$ Bulmer, ${ }^{1} \mathrm{~A}$ Nousheh-Moore, ${ }^{1} \mathrm{~V}$ Jones. ${ }^{1}$ Paediatric Department, North Middlesex University Hospital, London, UK; ${ }^{2}$ Aspiring Improvers, UCL Partners, London, UK

\subsection{6/archdischild-2020-rcpch.346}

Aims To develop a method of continuous assessment of subjective staff morale reporting and use the reporting data to inform improvements to staff wellbeing.

Methods A system, entitled MOODSEY, was developed asking multidisciplinary staff in a busy urban paediatric unit to anonymously retrospectively rate each shift as a 'Good Day' or 'Bad Day' at its conclusion. Broad demographic information was collected to aid with subgroup analysis. 'Complete' data required all 4 fields completed. This data was aggregated, and a score was plotted on a run chart.

Prior to data collection, a listening exercise occurred to generate themes that staff reported as affecting their shifts. When reporting, staff were encouraged to signal which of these themes had led to their choice. These themes were aggregated and analysed using the Model for Improvement to inspire changes that would improve future scores $\&$ the frequency of themes being reporting.

Paper and digital reporting techniques were designed to be minimally time-consuming and local champions encouraged reporting

Results The unit average MOODSEY score was plotted on a scale of -1 to +1 plotted on a run chart. In the first 2 months of reporting, a median score of 0.7 with statistically appropriate variability was found. Statistically significant shifts were seen for both positive and negative reporting.

Thematic data showed that negative reporting was most commonly associated with Poor Staffing, Incivility and Unsupportive Seniors whilst positive reporting was associated with a Good Atmosphere, Good Teamwork and Supportive Seniors.

885 responses were collected in total (mean: 12.5 responses/day). The presence of champions on shift improved response rate for all roles. $66 \%$ of reporting cards were completed fully.

Conclusion The MOODSEY system has proven the concept of 'smart real-time monitoring of staff morale'. Thematic analysis correlates with existing findings in the literature. Further iterations are required to integrate the project into the department however the encouraging initial success gives a platform for ongoing assessment of work aimed at staff wellbeing.

\section{G404(P) SEEING STARS - THE INTRODUCTION OF MOTIVATIONAL TRAINEE STAR CHARTS: A QUALITY IMPROVEMENT PROJECT}

F Dastur Mackenzie, E Charles. Paediatric Department, Croydon University Hospital, London, UK

10.1136/archdischild-2020-rcpch.347
The aim of our project was to introduce an initiative to support completion of supervised learning events (SLEs) and to encourage praise of trainees' successes with the goal of improving the quality of paediatric training and to boost trainee's morale.

All paediatric trainees are required by the $\mathrm{RCPCH}$ to collect a portfolio of various (SLEs) during their rotations, however finding the time to complete these assessments can be challenging. Furthermore, although there are systems in place for reporting error and malpractice within the NHS, there are few initiatives that promote the recognition of positive achievement.

We conducted a short questionnaire amongst paediatric trainees from ST1-ST8 looking at trainees' experiences in gaining SLEs, and on the effect of SLEs on trainee satisfaction. We found that the majority $(>50 \%)$ of trainees did not feel supported in gaining SLEs during their placement. We also found that the majority $(>70 \%)$ of trainees felt that receiving praise for successfully completing SLEs significantly affected their job satisfaction. Unfortunately, few trainees reported receiving this praise in practice.

We then introduced motivational trainee star charts. For ST1-ST8 trainee's stars were awarded for the completion of a variety of clinical procedures. For ST4-ST8 trainees and for consultants stars were also awarded for completing supervised clinical examinations, case-based discussions and direct observed procedures with junior trainees.

Following the intervention we carried out a further survey to analyse the impact of the star charts on trainee's motivation. The majority of trainees reported an increased number of SLEs gained per week following the intervention (88\%) and that they felt better supported to complete SLEs by seniors (88\%). There was an overall increase in job satisfaction $(100 \%)$ and it was reported that they found it easier to share opportunities with peers to perform skills (100\%).

Due to the success of the project the star charts were taken on by the department and were used for subsequent cohorts of trainees.

\section{G405(P) USING DIGITAL MEDIA TO IMPROVE ACCESS TO TEACHING}

${ }^{1} \mathrm{SM}$ Harris, ${ }^{2} \mathrm{~A}$ Javaid, ${ }^{2} \mathrm{SM}$ Constantinou. ${ }^{1}$ Paediatrics, Aneurin Bevan Heath Board, Newport, UK; ${ }^{2}$ Paediatrics, Noah's Ark Children's Hospital, Cardiff and Vale University Health Board, Cardiff, UK

\subsection{6/archdischild-2020-rcpch.348}

Method Uptake of traditional face-to-face MRCPCH written exam teaching sessions in Wales has been poor for three years despite interest being high among trainees. The aim of this project was to explore examinee experiences with the teaching programme and to use learner suggestions as part of a collaborative process to design a new teaching programme.

A survey was sent to doctors working in paediatrics in Wales. Data was collected from February until March 2019. Questions included quantitative information on exam teaching uptake as well as qualitative questions on suggestions for improvement. Results were then used to design a new teaching system.

Results There were 17 respondents. Reasons given for not attending teaching included having prior clinical commitments (47.1\%) or the teaching being held too far from their base hospital (11.8\%). The two most popular suggestions to 